\title{
MONOCULTURAS VERSUS ECOLOGIAS: POR UM PROTOCOLO INTERNACIONALIZADOR
}

Monocultures versus ecologies: on behalf of an internationalizing protocol

Monoculturas versus ecologías: por un protocolo internacionalizador

Received: july/2020

Accepted: august $/ 2020$

Available online: september/2020

Rubens Lacerda de Sá. Doutor em Linguística Aplicada, Instituto Federal de Educação, Ciência e Tecnologia de São Paulo (IFSP), Brasil

E-mail: rubens.sa@ifsp.edu.br

Resumo: A proposta deste ensaio é esboçar a pinceladas uma sugestão de protocolo futuro para ações afirmativas orientadas ao aprimoramento de programas de internacionalização. Desse modo, caminhos plurais, multissemióticos, axiológicos e ontologicamente orientados visando ao benefício ad aequitas dos atores imbricados nesses programas poderão ser criados sob a égide da epistemologia de Sousa Santos (2002). Trata-se de um recorte dos comentários e considerações finais de minha tese de doutorado.

Palavras-chave: Monoculturas, Ecologias, Internacionalização.

Abstract: The purpose of this essay is to brushstroke a suggestion of a future protocol for affirmative actions aimed at improving internationalization programs. Thus, plural, multi-semiotic, axiological and ontologically

RBPPD/BRJPD | Vol. 2 | n. 3 | p. 339-353, 2020. 
oriented paths aiming at the ad aequitas benefit of the actors involved in these programs can be created under the aegis of Sousa Santos' epistemology (2002). This is an excerpt from the comments and final considerations of my doctoral thesis.

Keywords: Monocultures, Ecologies, Internationalization.

Resumen: El propósito de este ensayo es hacer una sugerencia de un protocolo futuro para acciones afirmativas dirigidas a mejorar los programas de internacionalización. De esta manera, se pueden crear caminos plurales, multisemióticos, axiológicos y ontológicos orientados al beneficio ad aequitas de los actores involucrados en estos programas bajo los auspicios de la epistemología de Sousa Santos (2002). Este es un extracto de los comentarios y consideraciones finales de mi tesis doctoral.

Palabras clave: Monoculturas, Ecologías, Internacionalización.

\section{UM CONVITE À METÁFORA}

Ao abrir este ensaio penso que convém usar uma peça cinematográfica como uma metáfora que se alinha ao axioma que orientou a pesquisa que serviu de base. Logo, farei eventuais referências ao enredo do primeiro filme da trilogia The Matrix, dos irmãos Larry e Andy Wachowski (1999; 2003). Escolhi essa obra, pois, segundo Silva (2007), ela trata da relação do homem em busca do seu eu existencial que é encolhido, camuflado, silenciado e apagado sob o auspício da satisfação de supostas demandas macrossociais no bojo de um sistema de reprodução capitalista. Para tanto, na medida do possível, usarei a linguagem própria do cinema para orientar a dinâmica deste ensaio.

Isso posto, submeto, na acepção de Foucault (1969), meu texto ao escrutínio.

Je souhaite une agréable lecture à tous!

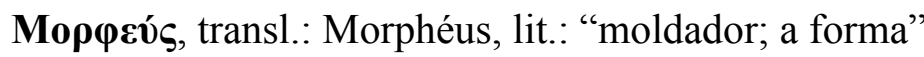

RBPPD/BRJPD | Vol. 2 | n. 3 | p. 339-353, 2020. 
Na mitologia grega, Morpheu era o deus dos sonhos que assumia variadas formas. Morava em uma caverna decorada com flores que continham um efeito sedativo e dormia em um leito da madeira de ébano. Diz-se que dentre suas habilidades estava a de possibilitar aos humanos a capacidade de produzir conhecimento enquanto adormecidos. Foi exatamente isso o que Morpheu, inadvertidamente, propiciou a Neo - a possibilidade de sair do mundo dos sonhos, da Matrix alucinógena. Ao sair da Matrix, irreal e virtual, Neo entra em estado de choque após conhecer o mundo real, sombrio e assustador. Fica horrorizado ao descobrir o que a Matrix ilusória faz com os seres humanos para manter um sistema baseado em uma alegoria (ideologias?!) que beneficiam uns poucos em detrimento da maioria.

Neo se dá conta, então, que o ser humano é usado na Matrix como fonte de energia, a força que movimenta as suas engrenagens. Entretanto, também percebe que os mesmos seres humanos que alimentam a Matrix podem reconfigurá-la e transformá-la rompendo com o ciclo de dominação, prejuízos e sofrimentos. Não obstante, para o idealizador da complexa Matrix, seu arquiteto, Neo não passa de uma anomalia, um vírus, porque continua sendo irremediavelmente humano, apesar de seus esforços de reinseri-lo na Matrix usando a lógica positivista, matemática. Contudo, ao conversar com o arquiteto, Neo entende que o que se considera uma anomalia, um vírus irremediavelmente humano, é na verdade uma questão de escolha pessoal: conformar-se ou não ao sistema, à Matrix ilusória.

São bem curiosos esses detalhes sobre o filme The Matrix. As informações sobre Morpheu me fazem lembrar de outra obra cinematográfica ${ }^{1}$ que relaciona o esconder-se em uma caverna e, sob efeito alucinógeno, produzir conhecimento de forma crítica e engajada

\footnotetext{
${ }^{1}$ Sociedade dos Poetas Mortos. Um filme americano de 1989, do gênero drama, dirigido por Peter Weir.
}

RBPPD/BRJPD | Vol. 2 | n. 3 | p. 339-353, 2020. 
socialmente. Alio isso ao entendimento de Neo de que ele não é uma anomalia, um vírus. Antes, porém, é um ser humano que tem a capacidade de fazer escolhas para não mais servir de fonte de manutenção do poder e do controle que a Matrix dominadora exerce sobre todos.

Penso que esse é exatamente o mesmo cenário, objetivo, compromisso e desafio do cientista popperiano e do pesquis-a-dor social ontologicamente orientado. (Sá, 2017) Esse cientista sensível também entra em estado de choque ao perceber com claridade as mazelas sociais a que é submetida a sociedade. Passa, por conseguinte, a trabalhar arduamente para identificar e romper com o controle que é exercido pelas ideologias dominantes e por aqueles que operam tais ideologias em favor da manutenção de seu poder e benefício próprios. Sua meta, como Neo no filme, passa a ser alertar, recrutar e libertar a tantos quanto for possível. Embora, esse cientista esteja ciente de que sua empreitada será complexa, árdua e longeva, está disposto a lutar contra um sem fim de inimigos ${ }^{2}$ que, inconscientemente, são manipulados pela Matrix.

\section{DO QUE ESTOU FALANDO?}

Há muitos outros detalhes ${ }^{3}$ no filme The Matrix que guardam relação com a pesquisa que deu origem a este ensaio. Mas, me falta o tempo e o espaço para continuar com a analogia. Meu objetivo foi indicar que a distopia que marca o filme também caracterizou a pesquisa. No entanto, tanto no filme, parcialmente inspirado na obra de Platão (2002), bem como na pesquisa, é possível o rompimento com o ciclo de dominação. Meu objetivo foi

\footnotetext{
${ }^{2}$ No filme, os Smith são caçadores de anomalias que ameaçam a Matrix e dos humanos que a alimentam

${ }^{3}$ A nave chamada Nabucodonosor, o paraíso chamado Zion, Cypher, o Oráculo, a estrangeiridade de Neo, etc.
}

RBPPD/BRJPD | Vol. 2 | n. 3 | p. 339-353, 2020. 
apontar os espaços para esse rompimento, bem como para o aprimoramento no programa de internacionalização para mobilidade acadêmica no lócus escolhido para a pesquisa e, ao mesmo tempo, sugerir rotas para melhorias.

Para tanto, embora use como âncora vários textos de Sousa Santos $(2000 ; 2007 ; 2010)$, atrevo-me a revisitar o texto Para uma Sociologia das ausências e uma Sociologia das emergências publicado em outubro de 2002 na Revista Crítica de Ciências Sociais, $n^{\circ}$ 63, por Boaventura de Sousa Santos. Meu objetivo foi fazer uma breve releitura do conceito esboçado nesse texto e sugerir o esboço, o rascunho de um protocolo de ações propositivas visando à hospitalidade derridiana aos estudantes internacionais.

Antes, porém, é preciso fazer uma breve consideração sobre o vocábulo protocolo para que eu possa situá-lo em meu texto. O fato é que, atualmente, essa palavra abrange um leque de significados que variam de atos formais públicos a critérios e procedimentos a serem seguidos, além de um conjunto de intenções para a realização de um pré-acordo. O objetivo de um protocolo é a eficiência na execução de certa ação ou alcance de um objetivo.

Etimologicamente $^{4}$, o substantivo protocolo surgiu em meados de 1540 a partir do Francês medieval, prothogall, com o sentido de "rascunho de um documento". No Latim, o termo protocollum significa literalmente "rascunho ou primeira folha de um manuscrito contendo as intenções e as erratas”. O vocábulo Grego, prōtokollon, confirma a ideia de rascunho de um manuscrito, i.e. prōtos "primeiro" + kolla "cola".

\footnotetext{
${ }^{4}$ Fontes: Dicionário Eletrônico, Copyright (C) 2005-2018 Apple Inc. Todos direitos reservados; Online Etymology Dictionary. Disponível em https://www.etymonline.com/search?q=Protocol Acesso 26/03/2019.
}

RBPPD/BRJPD | Vol. 2 | n. 3 | p. 339-353, 2020. 
Embora atualmente o uso do termo em pauta esteja associado à descrição dos procedimentos a serem adotados para a consecução de um ato, para os fins a que se destina este ensaio, o termo protocolo é associado a sua raiz etimológica, ou seja, como um "rascunho; um manuscrito contendo intenções" ou sugestões de ações para o desenho, concepção e formatação de um documento. Portanto, o que proponho aqui é um esboço, um rascunho para a sugestão de um protocolo de ações visando ao aprimoramento da política de internacionalização para mobilidade acadêmica no lócus da pesquisa. Assim, ao usar o termo protocolo estou me referindo a um rascunho ou esboço de possíveis ações.

\section{MONOCULTURAS VS. ECOLOGIAS: CONTRAPONTOS E O PROTOCOLO}

Isso posto, continuo com Boaventura de Sousa Santos que contrapõe, em seu texto, cinco monoculturas a cinco ecologias. Para o sociólogo (2002, p. 247), a lógica que opera essas monoculturas racionais objetiva "produzir não-existências, invisibilidade, desqualificação, ininteligibilidade e descarte de um modo irreversível”. Em contrapartida, as ecologias são epistemologicamente transgressoras, pois põe em cheque o modelo de produção das monoculturas totalitárias propondo "alternativas e inconformismo com o descrédito e a luta pela credibilidade" promovidos pela racionalidade. Procurarei estabelecer, então, um paralelo entre as monoculturas e ecologias de Sousa Santos com um rascunho, um esboço de um protocolo hospitaleiramente internacionalizador.

Protocolo um: A primeira monocultura é a do saber e do rigor do saber. Trata-se da criação de um “cânone exclusivo para a produção de conhecimento em que tudo o que não é legitimado aqui não é reconhecido e inexiste”. (p. 247) Os Programas de Internacionalização para Mobilidade Acadêmica no Brasil, doravante PIMAB, seguem, em minha percepção, um

RBPPD/BRJPD | Vol. 2 | n. 3 | p. 339-353, 2020. 
modelo estabelecido pelo eixo do norte global. Logo, há um esforço árduo para que se reproduza aqui o que já se faz lá.

Para contrapor esse modelo, os PIMAB devem aderir à ecologia de saberes, ou seja, "abrir a possiblidade de diálogo e disputa epistemológica entre saberes”. (p. 250) Isso implica à promoção de pluralidade de saberes e conhecimentos científicos e não-científicos. Sendo assim, a meta dos PIMAB deve ser a de propiciar intercâmbios, não apenas a suplantação de um saber hegemonicamente classificado.

Minha sugestão de protocolo é, portanto, que os estudantes internacionais não produzam conhecimento e contribuam para a pesquisa na universidade somente durante sua estada enquanto participantes dos PIMAB. Mas, que se mantenha um protocolo de produção e intercâmbio de saberes com os egressos com o fito de se identificar o impacto nas comunidades e nos países de origem desse alunado após sua participação em um PIMAB. Esse protocolo também servirá para a melhoria dos programas no sentido de que as pesquisas que se realizam em seu interior sejam, de fato, ontológicas e socialmente orientadas.

Protocolo dois: A segunda monocultura é a do tempo linear. Essa refere-se à construção de uma linha do tempo histórica dos "últimos 200 anos — progresso, revolução, modernização, desenvolvimento, crescimento e globalização". (p. 247) Em outras palavras, estabelece-se aqui uma relação temporal de assimetria entre o atrasado e o avançado. Isso é o que Koselleck (2006, p. 112) chama de “contemporaneidade do não contemporâneo”. No caso dos PIMAB, o que percebo é uma corrida maluca, do ponto de vista educacional, para chegada ao Olimpo da contemporaneidade. Não-ser-estar atrasado ou ser deixado para trás significa converter-se em apenas um resíduo na escala do tempo.

RBPPD/BRJPD | Vol. 2 | n. 3 | p. 339-353, 2020. 
A ecologia das temporalidades é o modelo que se opõe a essa lógica. Sua proposta é criar condições para a libertação de práticas sociais consideradas como residuais. Pensando nos PIMAB, isso significa valorizar, a cultura, os saberes e as habilidades locais que são trazidas pelos estudantes internacionais. Ou seja, "a presença ou relevância de diferentes culturas deixa de ser uma manifestação anacrónica de primitivismo para se tornar uma outra forma de viver a contemporaneidade". (p. 251)

Como segundo protocolo relacionado a essa ecologia pergunto: por que um estudante de um vilarejo distante na região altiplana da Bolívia ou do Perú, e que produz chuño de excelência, deveria apresentar ao seu povoado milenar técnicas agrícolas consideradas novas, modernas e high-tech para a produção em larga escala de um produto supostamente de melhor qualidade? Qual a lógica operante que determina o que é, para quem e para quê serve essa 'produção em larga escala' e um 'produto de melhor qualidade'?

Assim, penso que uma das aplicações possíveis para a ecologia das temporalidades é criar condições para troca de saberes. O que quero dizer, com a pergunta no parágrafo anterior, é propiciar que o estudante internacional traga à universidade que o acolhe seus saberes que podem ser considerados residuais e locais, mas que poderão contribuir para que os programas de internacionalização propiciem, ainda mais, uma relação simétrica e de ganhos a esses estudantes e suas comunidades de origem.

Protocolo três: A terceira monocultura é a da classificação social cuja meta é a da "naturalização da diferença e das hierarquias" por meio de "classificação racial, sexual, social", etc. (p. 247). Por meio dessa monocultura se estabelece a relação de dominação e a

RBPPD/BRJPD | Vol. 2 | n. 3 | p. 339-353, 2020. 
polarização de contratos sociais. Nos PIMAB, incorre-se no equívoco da promoção, ainda que inadvertida, de uma lógica colonialista cuja “conotação latente é de subalternidade”. (p. 250)

A ecologia dos reconhecimentos baseia-se nos pressupostos da etnografia crítica proposta por Sousa Santos (2001). Para ele, (2002, p. 252), essa consiste na "desconstrução tanto da diferença como da hierarquia", pois "as diferenças que subsistem quando desaparece a hierarquia tornam-se uma denúncia poderosa que a hierarquia exige para não desaparecer”.

Minha compreensão é que essa ecologia é a mais relevante para uma visão mais holística da dinâmica dos PIMAB. Convirjo aqui com o conceito de mímica de Homi Bhabha (2013) para assentir que o hemisfério sul busca a excelência alcançada pelo norte e o imita, não apenas no desenho e na formatação de seus programas de internacionalização, mas também na naturalização da alteridade e das hierarquias. Esse processo funda-se em preceitos colonialistas em que o Brasil, operando na lógica da monocultura do saber, repete com os países periféricos, aqui mesmo no sul, o mesmo feito pelos do norte em relação ao Brasil. Em outras palavras, o Brasil está para os países latinos que nos circunvizinham o que os Estados Unidos e a Europa estão para o Brasil. Assim, nossos PIMAB passam a ser vistos como referências ou fonte de saberes científico-acadêmicos.

Portanto, sugiro como protocolo o que propõe Sousa Santos (2002, p. 252) com a ecologia do reconhecimento, i.e. "a desconstrução tanto da diferença como da hierarquia". Em termos práticos isso implica desenhar políticas que contemplem as peculiaridades dos estudantes internacionais concedendo-lhes não apenas o acesso, mas um acolhimento nos termos discutidos na pesquisa que deu origem a este ensaio e, mais importante ainda, em acompanhamento socioacadêmico que propicie a esse alunado sucesso em sua estadia.

RBPPD/BRJPD | Vol. 2 | n. 3 | p. 339-353, 2020. 
Tais políticas devem ser marcadas pela clareza de objetivos e procedimentos, bem como ser desenhadas de modo a sistematizar todo o processo da hospitalidade. Os documentos que regem essas políticas institucionais devem estar disponíveis e de fácil acesso. Ademais, o site de internet, como uma das principais fontes de informação do programa institucional de internacionalização, deve estar disponível não somente em língua portuguesa e língua inglesa, mas também nas línguas dos maiores grupos de estudantes que aderem ao programa de mobilidade acadêmica da universidade.

Protocolo quatro: A monocultura da escala dominante é a quarta teorizada por Sousa Santos (2002). Nessa lógica os que não pertencem a essa escala, a dominante, são irrelevantes. Em tempos de globalização neoliberal quem for mais eficaz e mais eficientemente internacionalizar-se, mais bem qualificado e mais autorizado estará para gerir ações nessa escala dominante. O local é o não-existente; melhor é o do outro desde que seja global. Sousa Santos (2002, p. 249) diz que "o local é improdutivo", pois se trata "de formas sociais de inexistência porque as realidades que elas conformam estão apenas presentes como obstáculos em relação às realidades que contam como importantes, as globais”.

A ecologia das trans-escalas consiste em "desglobalizar o local e reglobalizá-lo de modo contra-hegemônico ampliando a diversidade das práticas sociais ao oferecer alternativas ao globalismo localizado". (p. 252) Assim, como sugerem Andreotti, et al. (2016), os PIMAB deveriam, então, e.g. estar aliados aos pressupostos epistemológicos da cartografia social crítica em que os atores sociais tentam afirmar identidades, territorialidades, imaginários sociais e vínculos afetivos que são ameaçados pelo projeto desenvolvimentista hegemônico.

RBPPD/BRJPD | Vol. 2 | n. 3 | p. 339-353, 2020. 
Em termos de protocolo, penso que os PIMAB deveriam minimamente ouvir com muito mais frequência o que têm a dizer os estudantes internacionais. Isso pode ser feito por meio de canais abertos e de fácil acesso, mas que ao mesmo tempo preservem a privacidade e a identidade desses estudantes. Desse modo, será possível evitar ora desconforto ora descontentamento que pode ser produzido no interior desses programas.

Protocolo cinco: A monocultura da lógica produtivista cujo crescimento econômico é a meta central e inquestionável é a quinta e última que abordo neste ensaio. No interior de alguns PIMAB que operam segundo essa lógica, tudo o que é estéril ou desqualificado profissionalmente não é produtivo e, portanto, de menos valia. Em palavras de Sousa Santos (2002, p. 249), nessa monocultura o que não é produtivo é considerado "ignorante, residual, inferior, e local".

Para contrapor a essa lógica, Sousa Santos propõe a ecologia da produtividade que é a mais controversa, pois objetiva romper com o paradigma capitalista de desenvolvimento econômico e acumulação de bens ad eternum, ou seja, ele (2002, p. 249) sugere que as experiências possam ser "libertadas dessas relações de produção e, por essa via, se tornem presentes”. O ideal, então, é que os PIMAB e suas políticas propiciem aos estudantes internacionais condições de autogerenciamento e de rompimento com as relações de subalternidade capitalista.

Uma maneira de se atingir essa meta é o que sugiro como último protocolo, a saber, a atenção ao domínio da língua do país que acolhe. Garantir o melhor domínio possível da língua acolhedora propiciará ao estudante internacional condições de transitar nas práticas sociais no meio acadêmico com mais serenidade, além de identificar e posicionar-se contra

RBPPD/BRJPD | Vol. 2 | n. 3 | p. 339-353, 2020. 
ações de preconceito, discriminação ou outras injustiças que podem ser perpetradas pela linguagem. Bizon (2013) descreve em seu texto a dificuldade de alguns estudantes internacionais que passaram por dissabores, frustrações, decepções devido ao alto índice de reprovação em disciplinas cursadas na universidade. Esse alto índice se deu em decorrência do empobrecido domínio da língua do país que os acolheu.

Assim, entendo que para que se rompa com essa lógica da monocultura produtivista de facilitar o acesso desses estudantes, mas não muni-los de condições materiais linguísticas para a permanência em seus programas, penso ser necessário um investimento linguístico ainda mais consistente e consciente nesses estudantes. Isso implicará em 'romper com o paradigma capitalista de desenvolvimento econômico' inerente à engrenagem acelerada do mundo globalizado.

Em termos práticos, o ensino de língua e a aferição do domínio linguístico desse alunado deve ser, como sugere Sousa Santos (2002, p. 253), “despensado, desresidualizado, desracializado, deslocalizado e desproduzido". Mais adiante, o autor complementa (p. 254) dizendo que é preciso "revelar a diversidade e a multiplicidade das práticas sociais e credibilizar esse conjunto por contraposição à credibilidade exclusivista das práticas hegemónicas". Essa deve ser a rota a ser seguida nesse assunto.

Iniciando um pretenso arremate a este ensaio, gostaria de salientar que as ações de hospitalidade institucionais precisam ser centradas não somente no acesso e acolhimento inicial, mas também em acompanhamento socioacadêmico contínuo dos discentes internacionais que integram os PIMAB. Para isso, é preciso que haja a sistematização de uma política de acompanhamento de tais discentes uma vez inseridos na universidade. Assim, 
haverá uma diminuição na possibilidade de se formatarem práticas discursivas que velam desigualdades no seio de programas de internacionalização.

Meu objetivo aqui não é insinuar falhas nas universidades brasileiras e nos seus competentes esforços para atrair estudantes e pesquisadores internacionais. Meu almejo neste brevíssimo ensaio foi destacar que a perseguição pelo oferecimento da hospitalidade que contemple as necessidades de todos os discentes internacionais pode resultar em maior excelência acadêmica. Desse modo, ecoando as palavras de Claudia Rocha (2012, p. 134), os PIMAB contribuirão para que se atenue "o estranhamento diante do que se mostra diferente e pela presença de incertezas, desconforto, e, até mesmo, rejeição a costumes e valores trazidos pelo encontro" com esses estudantes, além de estar ciente de que "as tão desejadas transformações são muito, frequentemente, processos tensos, difíceis e doloridos". Minha singela pretensão é provocar discussões e avanços em torno desse tema.

\section{ARREMATANDO A REFLEXÃO}

Chegando ao fim do fim, gostaria de ressaltar que o estabelecimento de um protocolo que seja internacionalizador, ipso facto, não é uma tarefa fácil. Ensaiei neste manuscrito e obviamente concluo com a sensação de que ainda há muito a ser lido, discutido, ouvido, entendido. Sobre esse tema, ainda é preciso abordar vários outros conceitos como, e.g. soberania, Estado-nação, justiça, direito, territorialidades, deslocamentos migratórios, e por aí vai. Ainda é preciso que surjam pesquisas que relacionem essa temática e aprofundem o estudo sobre hospitalidade em Jacques Derrida, Emmanuel Lévinas, Hannah Arendt, Vilen Flusser, Edmond Jabès, Alain Montandon e tantos outros que tratam desse tema e outros

RBPPD/BRJPD | Vol. 2 | n. 3 | p. 339-353, 2020. 
correlatos, e.g. Michel Foucault, Norman Fairclough, etc. Há que se entender melhor essa grande coisa chamada internacionalização, bem como sua relação com ideologias.

Enfim, percebo que urge estudar, compreender, pesquisar, teorizar, agir, etc. Esse é o fado do pesquis-a-dor social. Essa é minha sina. Para desligar meu computador proponho a criação da sintaxe da hospitalidade, a sintaxe do deslocado, a sintaxe do desterritorializado, a sintaxe do ser e do ente (para não deixar Heidegger de fora!).

Aí está uma ótima proposta: meu próximo texto será sobre sintaxe!

\section{REFERÊNCIAS}

ANDREOTTI, V. O. et al. Internacionalização da Educação brasileira: possibilidades, paradoxos e desafios. In: LUNA, J. M. F. (Org.) Internacionalização do currículo: Educação, interculturalidade e cidadania global. Campinas, SP: Pontes, 2016, pp. 129-154.

BHABHA, H. K. O local da cultura. Trad. Myriam Ávila, Eliana Lourenço de Lima Reis e Gláucia Renata Gonçalves. 2 ed. Belo Horizonte: Editora UFMG, 2013.

BIZON, A. C. Narrando o exame Celpe-Bras e o programa PEC-G: a construção de territorialidade em tempos de internacionalização. $445 \mathrm{f}$. Tese (Doutorado). Campinas, SP: IEL/Universidade Estadual de Campinas, 2013.

FOUCAULT, M. L’archéologie du savoir. Paris: Gallimard, 1969.

KOSELLECK, R. Futuro passado: contribuição à semântica dos tempos históricos. Trad. Wilma Patrícia Maas, Carlos Almeida Pereira. Rev. César Benjamin. Rio de Janeiro: Contraponto, 2006.

PLATÃO. República. Trad. Enrico Corvisieri. Rio de Janeiro: Ed. Best Seller, 2002.

ROCHA, C. H. Reflexões e propostas sobre língua estrangeira no ensino fundamental I: plurilinguismo, multiletramentos e transculturalidade. Campinas, SP: Pontes Editores, 2012.

SÁ, R. L. Imigrantes hispano-americanos, (inter)culturalidade crítica e língua portuguesa. Revista Estudos Acadêmicos de Letras. Centro de Estudos e Pesquisas em Linguagem e Centro de Estudos e Pesquisas em Literatura da Universidade do Estado de Mato Grosso. v. 10, $\mathrm{n}^{\mathrm{o}} 01$, Julho, 2017.

SILVA, F. C. The Matrix: a aventura da formação no mundo tecnologizado. Educação e Sociedade. Campinas, vol. 28, n. 101, set./dez. 2007, pp. 1545-1561.

SOUSA SANTOS, B. Um discurso sobre as ciências. 5a ed. São Paulo: Cortez, 2000.

RBPPD/BRJPD | Vol. 2 | n. 3 | p. 339-353, 2020. 
SOUSA SANTOS, B. Nuestra America: reinventing a subaltern paradigm of recognition/redistribution. Theory Culture and Society, 18(2-3), 2001.

SOUSA SANTOS, B. Para uma Sociologia das ausências e uma Sociologia das emergências. Revista Crítica de Ciências Sociais, 63, Outubro, 2002, pp. 237-280.

SOUSA SANTOS, B. Para além do pensamento abissal: das linhas globais a uma ecologia de saberes. Novos Estudos CEBRAP 79, novembro, 2007, pp. 71-94.

SOUSA SANTOS, B.; MENESES, M. P. (Orgs.). Epistemologias do sul. São Paulo: Cortez, 2010. 